\title{
INCIDENTAL FINDINGS DURING FOLLOW-UP SCANS IN ONCOLOGICAL PATIENTS
}

\author{
COSMIN CARAIANI ${ }^{1}$, ANAMARIA POP ${ }^{2}$, ADRIANA CALIN ${ }^{3}$, \\ LIDIA CIOBANU ${ }^{2,4}$, CLAUDIA MILITARU ${ }^{5}$, ALEXANDRA BERGHE ${ }^{6}$, \\ RADU BADEA ${ }^{1,2}$
}

\author{
${ }^{1}$ Medical Imaging Department, Iuliu Hatieganu University of Medicine and \\ Pharmacy, Cluj-Napoca, Romania \\ ${ }^{2}$ Gastroenterology and Hepatology Department, Regional Institute of \\ Gastroenterology and Hepatology, Cluj-Napoca, Romania \\ ${ }^{3}$ Radiology Department, Regional Institute of Gastroenterology and Hepatology, \\ Cluj-Napoca, Romania \\ ${ }^{4}$ Gastroenterology and Hepatology Department, Iuliu Hatieganu University of \\ Medicine and Pharmacy, Cluj-Napoca, Romania \\ ${ }^{5}$ Oncology Department, Medisprof, Cluj-Napoca, Romania \\ ${ }^{6}$ Medical Informatics and Biostatistics Department, Iuliu Hatieganu University of \\ Medicine and Pharmacy, Cluj-Napoca, Romania
}

\section{Abstract}

Background and aim. To assess the prevalence of incidental findings during follow-up scans of patients with oncologic pathology.

Methods. 499 follow-up scans from different patients with cancer pathology were retrospectively analyzed. Findings which were not suspected by the clinician or known from previous scans were considered as incidental lesions. We excluded lesions that were already suspected by the clinician or were already recorded in the patient's history before the initial computed tomography (CT) scan. The CT scans were performed on two different machines ("Siemens Somatom Sensation, 64 slices, Erlangen, Germany" and "Siemens Somatom Emotion, 16 slices, Erlangen, Germany").

Most of the patients had a native scan followed by a thoraco-abdominal-pelvic image acquisition after the injection of intravenous contrast media.

Results. $28 \%$ of the patients had unsuspected incidental findings. The prevalence of incidental findings was similar: $56.6 \%$ of them were found in men and $43.4 \%$ in female patients. In 6 cases (1.2\%) the presence of unsuspected pulmonary embolism was discovered. From these cases, 5 (83.3\%) had metastatic disease at the moment of the follow-up CT scan and 1 (16.6\%) had metastasis-free disease. In 17 patients out of $499(3.4 \%)$ we incidentally made an important discovery which either changed the cancer therapy or required immediate treatment.

Conclusion. Incidental findings are not rare in oncological patients and the radiologist has to be aware of their presence in order not to overlook them and to correctly diagnose them.

Keywords: pulmonary embolism, cancer, contrast-enhanced computed tomography, follow-up 


\section{Introduction}

Patients with oncological diseases benefit from the development of modern imaging techniques like contrastenhanced computed tomography (CECT). In order to assess the extent of the disease and to evaluate its response to treatment, cancer patients undergo routine CECT studies every 3 months, 6 months or 1 year [1].

The extensive use of modern imaging techniques led to an increasing number of incidental findings, which have to be differentiated from oncologic relevant findings and correctly interpreted.

Incidental pulmonary embolism (PE) in cancer patients is increasingly encountered, varying between $2.6 \%$ and $3.4 \%$ in the literature $[2,3]$ with pancreas, ovary and brain cancers being the most frequent causes for thrombotic complications [4].

Identification and correct reporting of PE is crucial for cancer patients because it represents a substantial cause of morbidity and mortality. Most cases of death from PE occur in patients with unsuspected and therefore untreated disease $[5,6]$.

Another crucial aspect is to differentiate incidental lesions from oncologic relevant findings. Misinterpretation of benign adrenal or lung nodules as secondary lesions may lead to over-staging and subsequent changes in the patient's therapy.

\section{Material and methods}

Any lesion not suspected or recorded in the patient's medical history was classified as incidental finding.

\section{Patients}

In the present study, 499 CT scans of cancer patients were retrospectively analyzed.

Medical history was provided by the oncologist for all the patients included in the study.

\section{Inclusion criteria}

Patients with known malignancies who underwent initial CT evaluation or follow-up scans, indicated by their referring physician, were included in the group. Findings which were not suspected by the clinician or known from previous scans were considered as incidental lesions. In the case of chronic pathology (gallstones, urolithiasis), the patient's history was taken into account.

\section{Exclusion criteria}

We excluded lesions that were already suspected by the clinician or were already recorded in the patient's history before the initial CT scan.

Patients with respiratory symptoms and clinically suspected pulmonary embolism, confirmed by the radiologist (4 patients) were also excluded from the study - only patients with unsuspected and incidentally found pulmonary embolism remained in the group.

Pulmonary nodules were excluded from the study, due to the lack of specificity of CT in defining their nature and also common liver focal lesions like biliary cysts or hemangiomas or cortical renal cysts (as they present no clinical relevance).

\section{CT Protocol}

The CT scans were performed on two different CT machines ("Siemens Somatom Sensation, 64 slices, Erlangen, Germany" and "Siemens Somatom Emotion, 16 slices, Erlangen, Germany"). The CT protocol was not uniform as it was adapted to the patient's pathology and their creatinine values (patients with creatinine clearance lower than $30 \mathrm{ml} / \mathrm{min}$ calculated after the Cockcroft-Gault Equation were not injected with intravenous (i.v.) contrast media). Oral contrast media was administrated in most patients, except for those with renal malignancies.

In most cases, the CT scans included in the acquisition thorax, abdomen and pelvis but in some cases, at the oncologist's demand, pelvis was not included in the scan.

After contrast media injection, a single phase acquisition, 60 seconds after contrast media injection or a double phase acquisition, non-enhanced and enhanced at 60 seconds after injection of contrast media, were performed. In some cases, arterial phases or late phases of the upper abdomen were also performed, in order to characterize focal liver lesions (FLL).

10 of the patients had clinically suspected pulmonary embolism at the moment of the follow-up CT scan and therefore a thoracic angio-CT scan was performed in order to better visualize the pulmonary arteries. Pulmonary embolism was confirmed in 4 of these patients.

\section{Image analysis}

The following data were recorded for each patient: sex, age, tumor type (there were 8 cases of synchronous tumors and 5 cases of patients with unknown primary tumor), presence of unsuspected pulmonary embolism, presence and characterization of other incidental findings, presence of metastatic disease. Images were initially interpreted by one radiologist and then reinterpreted by two radiologists in consensus, for inclusion in the study.

The presence of incidental pulmonary embolism and other incidental findings were comparatively analyzed in patients with or without the presence of metastatic disease.

\section{Statistical Analysis}

Obtained data were descriptively analyzed. For each of the variables used we calculated the frequencies to assess the distribution of patients based on the analyzed characteristics. Except for the age of the patient, all other variables were qualitative or binary.

\section{Results}

The total number of tumors was 510 as 11 patients had synchronous neoplasms. All patients had a history of an oncologic disease and underwent initial evaluation scans or follow-up scans as indicated by the oncologist, between January and August 2015.

The malignancies encountered in our group are summarized in Table I. 
Table I. The distribution of oncologic pathology.

\begin{tabular}{|c|c|c|}
\hline Type of neoplasia & Nr Cases & $\%$ \\
\hline pulmonary & 79 & $15.49 \%$ \\
\hline prostate & 21 & $4.12 \%$ \\
\hline bladder & 33 & $6.47 \%$ \\
\hline stomach & 29 & $5.69 \%$ \\
\hline ovary & 26 & $5.10 \%$ \\
\hline renal & 53 & $10.39 \%$ \\
\hline breast & 68 & $13.33 \%$ \\
\hline colon & 73 & $14.31 \%$ \\
\hline rectum & 40 & $7.84 \%$ \\
\hline sarcoma & 6 & $1.18 \%$ \\
\hline endometrial & 5 & $0.98 \%$ \\
\hline pancreas & 15 & $2.94 \%$ \\
\hline testicles & 9 & $1.76 \%$ \\
\hline pharynx & 2 & $0.39 \%$ \\
\hline melanoma & 14 & $2.75 \%$ \\
\hline cervix of uterus & 5 & $0.98 \%$ \\
\hline salivary gland & 1 & $0.20 \%$ \\
\hline tongue & 1 & $0.20 \%$ \\
\hline ampulla of Vater & 3 & $0.59 \%$ \\
\hline frontal sinus & 1 & $0.20 \%$ \\
\hline lymphoma & 4 & $0.78 \%$ \\
\hline ureter & 5 & $0.98 \%$ \\
\hline penis & 2 & $0.39 \%$ \\
\hline gallbladder & 1 & $0.20 \%$ \\
\hline pleural mesothelioma & 1 & $0.20 \%$ \\
\hline skin & 1 & $0.20 \%$ \\
\hline thyroid & 1 & $0.20 \%$ \\
\hline neuroendocrine & 2 & $0.39 \%$ \\
\hline vaginal & 1 & $0.20 \%$ \\
\hline neck & 1 & $0.20 \%$ \\
\hline pelvic & 1 & $0.20 \%$ \\
\hline mesenteric & 1 & $0.20 \%$ \\
\hline unknown primary & 5 & $0.98 \%$ \\
\hline Total & 510 & $100.00 \%$ \\
\hline
\end{tabular}

The study group consisted of 57\% men and $43 \%$ women. The average age of the group was 62.17 years, while most of them had the age of 65 . The youngest patient was 25 years of age at the time of analysis, while the oldest was 93. Half of the sample was 63 years old or more. 11 patients had synchronous tumors and 5 had histologyproven metastases with unknown primary.

Frequency of unsuspected pulmonary embolism

In 6 cases $(1.2 \%)$ the presence of unsuspected pulmonary embolism was discovered (Figure 1). From this cases, $5(83.3 \%)$ had metastatic disease at the moment of the follow-up CT scan and $1(16.6 \%)$ had metastatic-free disease.

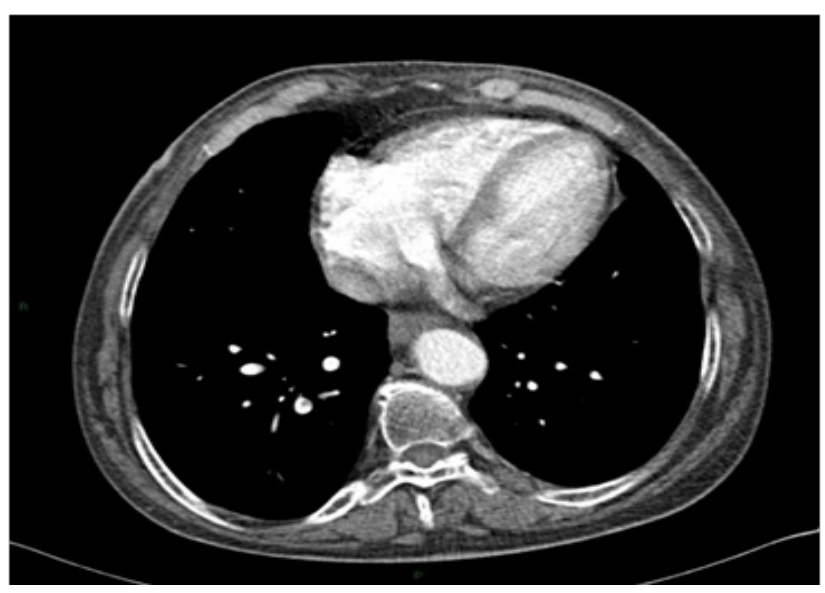

a

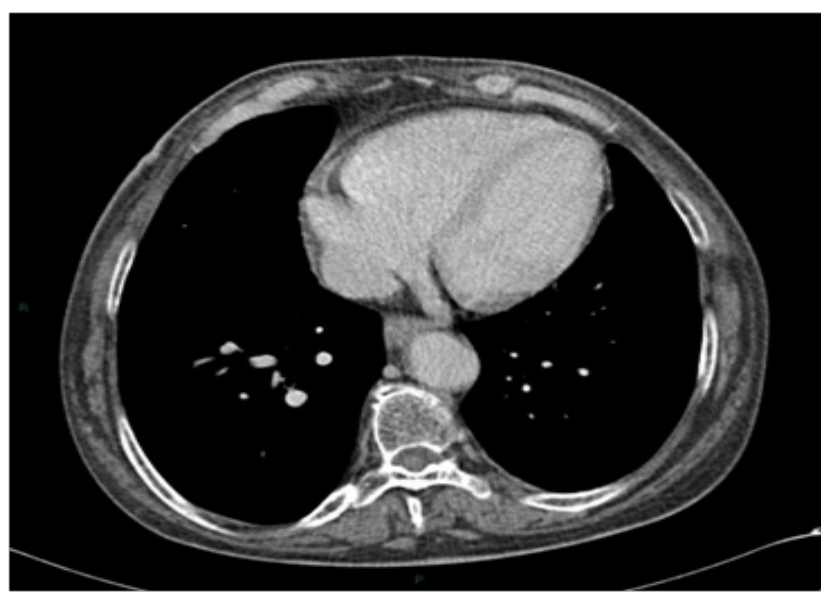

b

Figure 1. a-b. Pulmonary embolism on a segmental artery in the right lower lobe- clearly seen on the scan realized in the arterial phase (a) but very difficult to diagnose on the image obtained at the same level in the porto-venous phase (b).

The malignancies associated with pulmonary embolism were: gastric ( 2 cases), pancreatic ( 1 case), colon and syncronous ovary tumors (1 case), biliary cancer (1 case).

Frequency of unsuspected incidental findings (other than pulmonary embolism)

$28 \%$ of the patients had unsuspected incidental findings. We found 147 unsuspected findings in 139 patients ( 6 patients had 2 unsuspected findings and one had three unsuspected findings). The prevalence of incidental findings was similar: $56.6 \%$ of them were found in men and $43.4 \%$ in female patients.

The most frequent incidental findings were adrenal nodules (which can be confidently characterized as adenomas on the basis of the CT findings) and non- 
obstructive urolithiasis. Both adrenal nodules and urolithiasis were present in 24 patients (4.8\%). Gallstones were encountered in 15 of the patients $(3 \%)$.

All incidental findings are summarized in Table II.

Table II. Other incidental findings during follow-up scans.

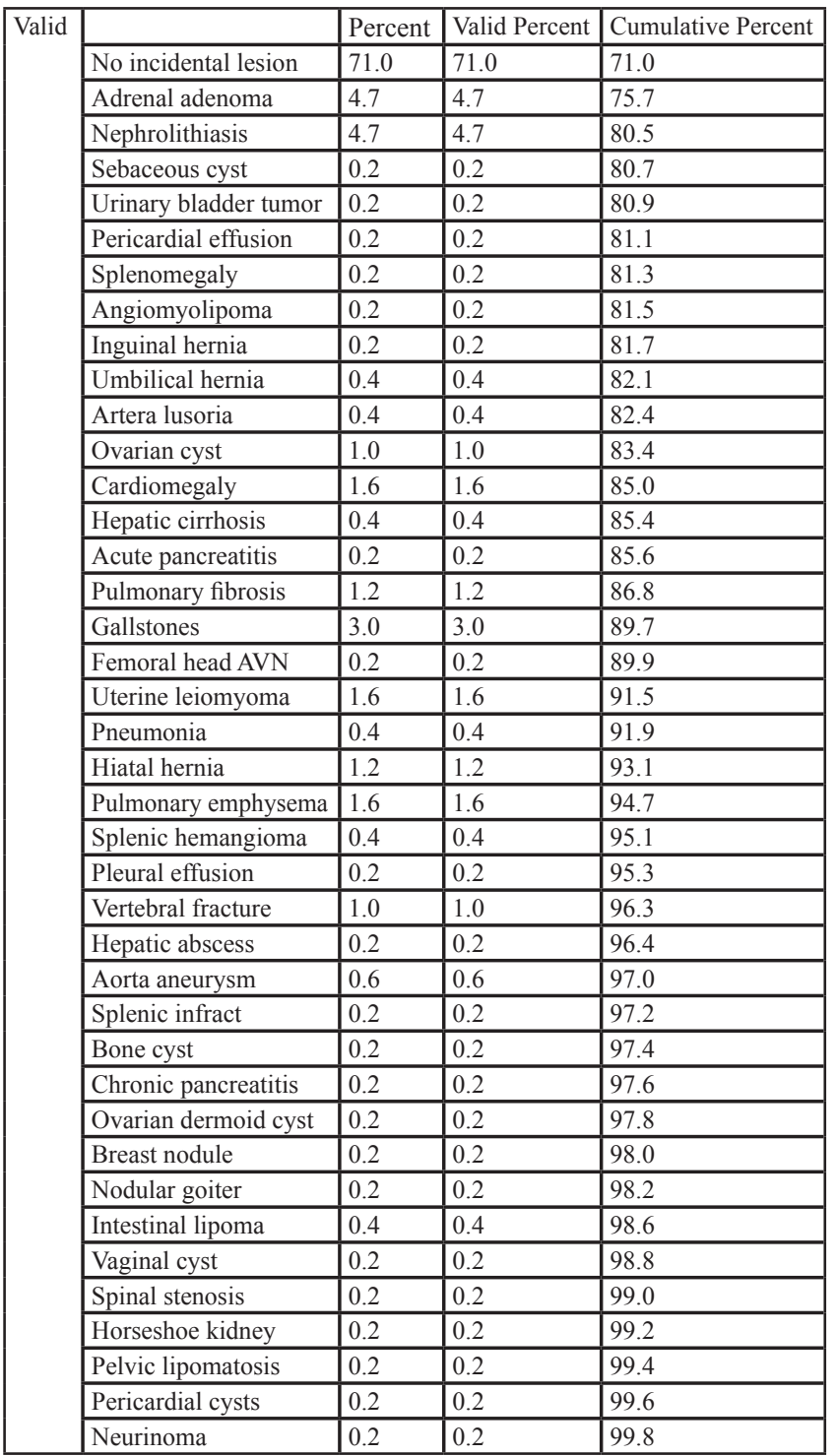

\section{Discussion}

Pulmonary embolism is a fearsome complication in cancer patients, potentially lethal if left untreated. Cancer patients with associated thrombotic events have a lower survival rate that those without thrombosis [7], the 1-year survival rate in cancer patients with associated thrombotic events reaching only $12 \%$ compared to $36 \%$ in patients without thrombosis [8]. This may be explained by the mortality due to thrombotic complications itself, but also to the fact that thrombotic complications mostly occur in patients with advanced disease. In our study 5 out of 6 patients $(83.3 \%)$ with unexpected, incidentally discovered pulmonary embolism had metastatic disease. All 4 patients with clinically suspected pulmonary embolism had, at the moment of the thrombotic event, metastatic disease.

Also, studies report that after experiencing an initial embolic event a patient may be at risk for circulatory collapse due to right-sided heart failure and a subsequent embolism may be fatal- which explains the high mortality of pulmonary embolism (PE) in patients with paraneoplastic hypercoagulability [9].

In our study incidental pulmonary embolism had a lower prevalence than previously described in the literature $(1.2 \%$ vs $2.6-3.4 \%)[10,11]$. A possible explanation for this fact would be that we did not include patients with clinically suspected PE $(n=4)$ in our study group. If we added these cases, the incidence of PE in our series would reach $2 \%$, close to data reported in the literature. Also in our group we had a high number of urinary tract and prostate cancers $(n=105,21 \%$ of all patients) which are rarely complicated by pulmonary embolism. The incidence of pulmonary embolism in patients with renal cancer is $0.5 \%$, most of them consisting of tumoral and non-thrombotic emboli $[12,13]$.

According to the literature data, in our study the malignancies associated with pulmonary embolism were pancreatic and ovary, but also we found PE in patients with gastric, biliary and rectal tumors [4].

As we try to reduce the radiation dose as much as possible, our protocol usually includes a single, venousphase acquisition of the thorax (Figure 2). Due to this protocol, which is not especially suited for detection of pulmonary embolism, some other cases of PE may be missed. Visualization of the main pulmonary trunk and of the left and right main pulmonary arteries was considered as satisfying by the radiologists who performed the image interpretation in our study. Nevertheless, PE limited to segmentary and subsegmentary arteries may be missed if a proper angiographic phase is not assessed. 139 patients $(27.8 \%)$ had other incidental findings. The amount of patients with incidental findings may be high due to the fact that the patients in our study group belong to an elderly age interval (mean age $=62.17$ years). Also a limitation of our study is the fact than we had access only to medical documents and patient history provided by the oncologist where, maybe, some lesions were not reported and registered, therefore we may over-estimated the frequency of incidental findings.

The most frequently encountered incidental finding was the presence of unilateral or bilateral adrenal nodules. It is of crucial importance to correctly determine the benign nature of an adrenal nodule in order to avoid overstaging of the oncologic disease, especially in patients with no other metastatic lesions, as the presence of an adrenal metastasis may hinder a curative treatment and influence the survival rate [14]. 


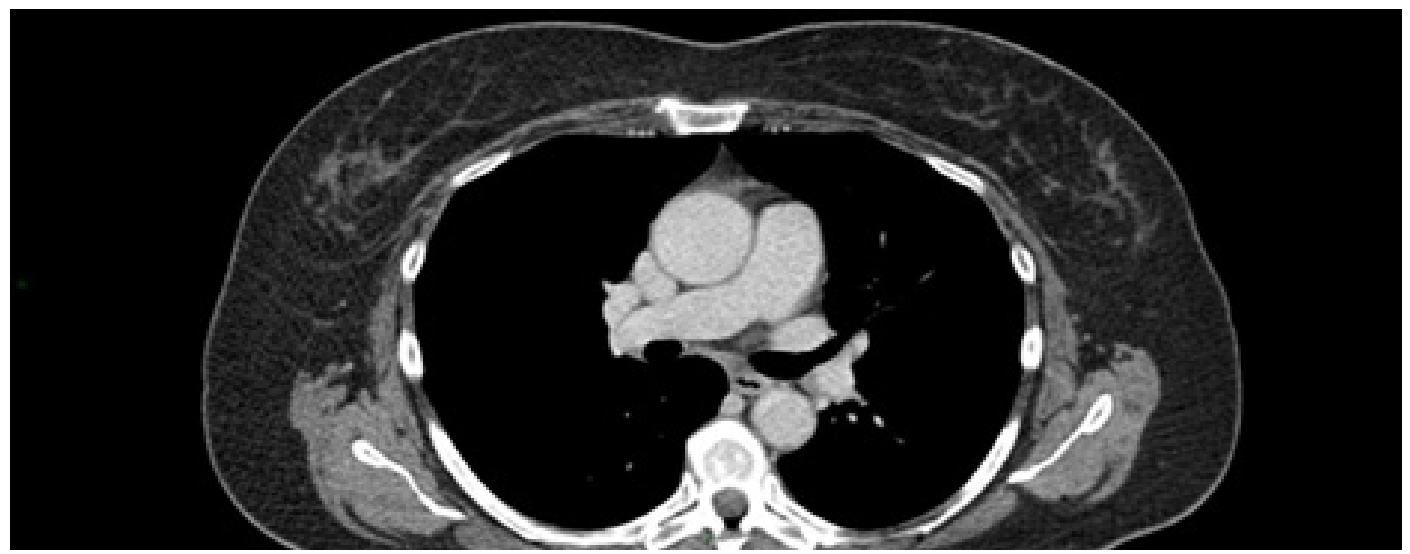

Figure 2. Pulmonary artery as seen at 60 seconds after injection of contrast media. Pulmonary embolism can be seen on main and lobar pulmonary arteries, but it's difficult to diagnose if located on segmental or subsegmental branches.

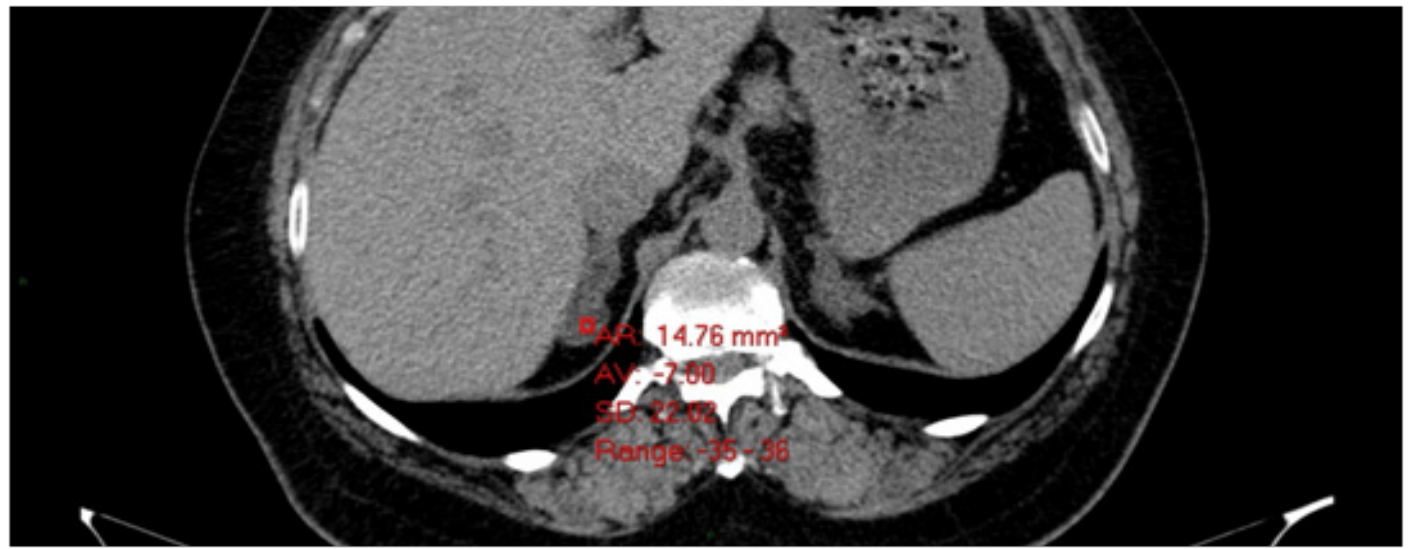

Figure 3. Adrenal lipid-rich adenoma with a negative mean density- suggestive for the presence of fat inside the lesion.

The incidental discovery of adrenal nodules on abdominal $\mathrm{CT}$ is as high as $5 \%[15,16]$. We differentiate adrenal adenomas from metastases by measuring the lesions density on non-enhanced CT scans- a lesion with attenuation values equal or below 10 Units of Hounsfield (UH) can be considered a lipid-rich adrenal adenoma (Figure 3), with a sensitivity of $71 \%$ and a specificity of $98 \%$ [17]. A sensitivity of $71 \%$ means that nearly $30 \%$ of benign adrenal nodules cannot be confidently characterized as benign by using this particular feature. Adrenal lesions which remain indeterminate after analysis of attenuation on non-enhanced CT scans can be further characterized by calculating the wash-out after injection of contrast media. It is considered that, on a 15 minutes delay acquisition, an absolute wash-out higher than $60 \%$ has a sensitivity of $86-88 \%$ and specificity of $92-96 \%$ for the diagnosis of adenoma $[18,19]$. If the nature of an adrenal lesion is still indeterminate, even after contrast media injection and wash-out calculation, followup scans can indicate the probable benignity of the lesionit is very rare for an untreated malignant lesion to remain dimensionally stable after a 6 months period [20]. Also, adrenal lesions need to be interpreted accordingly to the location of the primary tumor: for instance, a digestive tract tumor will very rarely cause adrenal metastases without causing liver metastases first. Other imaging techniques, such as MRI with in- and out-of-phase sequences or PET$\mathrm{CT}$ can be used to assess the nature of adrenal lesions.

We did not include pulmonary nodules in our study due to the lack of specificity of CT in characterizing them. The presence of benign pulmonary nodules can frequently lead to overstaging of the disease. There are some features which can indicate the benign nature of a pulmonary nodule. Specific calcification patterns (diffuse, laminated, central, popcorn) in a pulmonary nodule indicate a benign sequellar character. Clustering of multiple small nodules in a single location indicates an infectious origin. Small, irregular, subpleural opacities are generally due to scarring and ovoid circumscribed nodules adjacent to pulmonary fissures more likely represent intrapulmonary lymph nodes (Figure 4) $[21,22,23]$. Several studies proved that the probability of malignancy for a pulmonary nodule in a patient with colorectal carcinoma is ranged from $6.3 \%$ to $16.6 \%[24,25,26,27]$. It is considered that the presence of indeterminate PNs should not lead to a change of the therapeutic plan in colorectal cancer [27]. Due to the lack of specificity of $\mathrm{CT}$ in assessing the nature of PNs, we consider that the presence of indeterminate PNs should not influence the therapeutic plan in any type of primary cancer. 


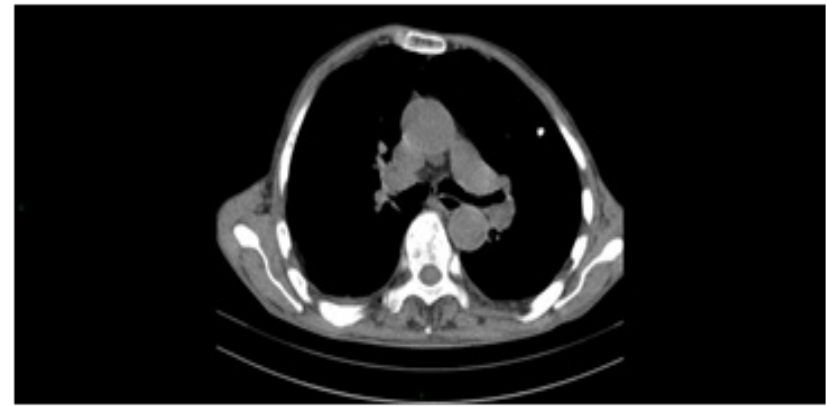

a

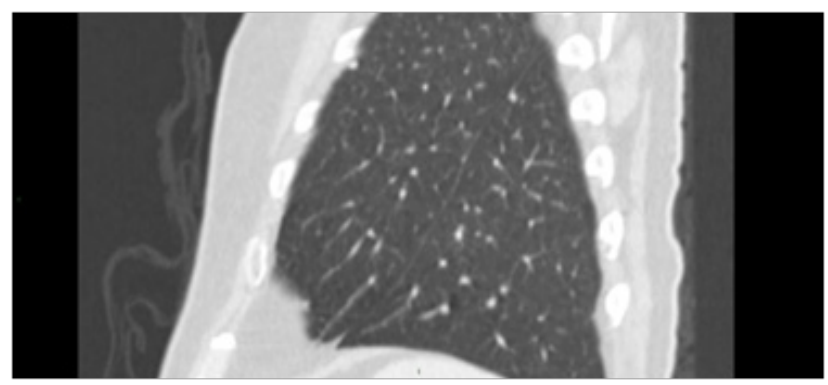

c

Figure 4. a-d. Radiologic aspect suggesting benign pulmonary nodules . Calcified node (a). Small subpleural nodule with an ovoid appearance and a prolongation which reaches the pleura (b). Ovoid node close to the oblique fissure more likely representing an intrapulmonary lymph node (c). A cluster of nodules, more likely of benign nature. After one month there was complete resolution of the nodules; we consider them more likely of infectious origin (d).

In 17 patients out of 499 (3.4\%) we incidentally made an important discovery which either changed the cancer therapy or required immediate treatment. The 6 cases with incidental pulmonary embolism were also included in this category. Besides incidental PE, we discovered one case of synchronous cancer (urinary bladder cancer in a patient followed up for pulmonary neoplasm), 2 cases of liver cirrhosis (both of them proved to be of alcoholic origin with negative viral tests), one case of acute pancreatitis, one case of avascular necrosis of the femoral head, 2 cases of acute pneumonia, 1 case of liver abscess (in a patient with no history of liver, biliary tract or pancreatic surgery or neoplastic lesions in this particular locations) and 3 cases of uncomplicated abdominal aortic aneurysms.

\section{Conclusions}

Incidental findings are frequently encountered in cancer patients.

Adrenal adenomas are one of the most frequently discovered incidental lesions and differentiating them from adrenal metastases is crucial for the further management of the patient.

In a minority of patients, incidental findings are clinically relevant and may lead to emergency treatment or changes in the patient's treatment plan.

\section{References}

1. Gosselin MV, Rubin GD, Leung AN, Huang J, Rizk NW. Unsuspected pulmonary embolism: prospective detection on
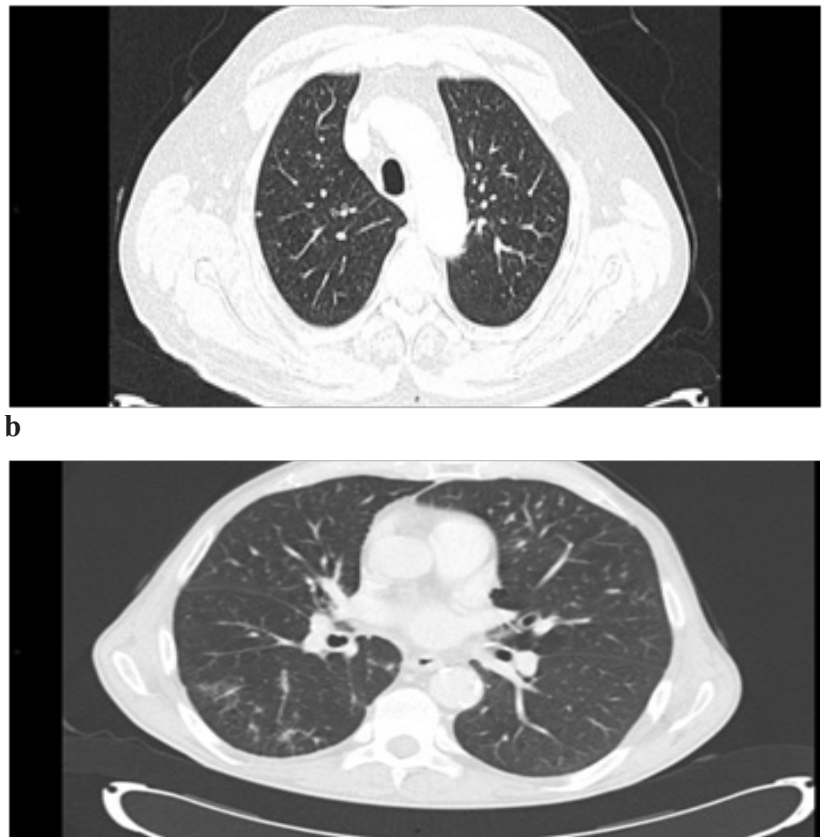

d routine helical CT scans. Radiology. 1998;208:209-215.

2. Dentali F, Ageno W, Becattini C, Galli L, Gianni M, Riva N, et al: Prevalence and clinical history of incidental, asymptomatic pulmonary embolism: a meta-analysis. Thromb Res. 2010;125:518-522.

3. Gladish GW, Choe DH, Marom EM, Sabloff BS, Broemeling LD, Munden RF. Incidental pulmonary emboli in oncology patients: prevalence, CT evaluation, and natural history. Radiology. 2006;240:246-255.

4. Lee AY, Levine MN. Venous thromboembolism and cancer: risks and outcomes. Circulation. 2003;107(23 Suppl 1):I17-I21.

5. Ryu JH, Olson EJ, Pellikka PA. Clinical recognition of pulmonary embolism: problem of unrecognized and asymptomatic cases. Mayo Clin Proc. 1998;73:873-879.

6. Pineda LA, Hathwar VS, Grant BJ. Clinical suspicion of fatal pulmonary embolism. Chest. 2001;120:791-795.

7. Cronin CG, Lohan DG, Keane M, Roche C, Murphy JM. Prevalence and significance of asymptomatic venous thromboembolic disease found on oncologic staging CT. AJR Am J Roentgenol. 2007;189:162-170.

8. Sørensen HT, Mellemkjaer L, Olsen JH, Baron JA. Prognosis of cancers associated with venous thromboembolism. N Engl J Med. 2000;343:1846-1850.

9. Wittram C, Maher MM, Yoo AJ, Kalra KM, Shepard J, McLoud TC. CT angiography of pulmonary embolism: diagnostic criteria and causes of misdiagnosis. Radiographics. 2004;24:1219-1238. 10. Hall WB, Truitt SG, Scheunemann LP, Shah SA, Rivera MP, Parker LA, et al. The prevalence of clinically relevant incidental findings on chest computed tomographic angiograms ordered to diagnose pulmonary embolism. Arch Intern Med. 2009;169:19611965.

11. Singh R, Sousou T, Mohile S, Khorana AA. High rates 
of symptomatic and incidental thromboembolic events in gastrointestinal cancer patients. J Thromb Haemost. 2010;8:18791881.

12. Parmar R, Bastola S, Adamo J. Renal Cell Carcinoma Presenting as Pulmonary Embolism in a Patient with HematuriaManagement Challenges. JSM Clin Oncol Res. 2015;3(1):10451046.

13. Abel E. Outcomes of RCC patients with venous thrombus presenting with pulmonary embolism: A multicenter analysis of 782 consecutive patients. Genitourinary Cancers Symposium. $2013 ; 433$.

14. Mitchell IC, Nwariaku FE. Adrenal masses in the cancer patient: surveillance or excision. Oncologist. 2007;12:168-174.

15. Bovio S, Cataldi A, Reimondo G, Sperone P, Novello $\mathrm{S}$, Berruti A, et al. Prevalence of adrenal incidentaloma in a contemporary computerized tomography series. J Endocrinol Invest. 2006;29:298-302.

16. Song JH, Chaudhry FS, Mayo-Smith WW. The incidental adrenal mass on CT: prevalence of adrenal disease in 1,049 consecutive adrenal masses in patients with no known malignancy. AJR Am J Roentgenol. 2008;190:1163-1168.

17. Boland GW, Lee MJ, Gazelle GS, Halpern EF, McNicholas MM, Mueller PR. Characterization of adrenal masses using unenhanced CT: an analysis of the CT literature. AJR Am J Roentgenol. 1998;171:201-204.

18. Caoili EM, Korobkin M, Francis IR, Cohan RH, Platt JF, Dunnick NR, et al. Adrenal masses: characterization with combined unenhanced and delayed enhanced CT. Radiology.
2002;222:629-633.

19. Korobkin M, Brodeur FJ, Francis IR, Quint LE, Dunnick NR, Londy F. CT time-attenuation washout curves of adrenal adenomas and nonadenomas. AJR Am J Roentgenol. 1998;170:747-752.

20. Dunnick NR, Korobkin M. Imaging of adrenal incidentalomas: current status. AJR Am J Roentgenol. 2002;179:559-568.

21. Yano $M$, Arai $T$, Inagaki $K$, Morita $T$, Nomura $T$, Ito $H$. Intrapulmonary satellite nodule of lung cancer as a $\mathrm{T}$ factor. Chest. 1998;114:1305-1308.

22. Byers TE, Vena JE, Rzepka TF. Predilection of lung cancer for the upper lobes: an epidemiologic inquiry. J Natl Cancer Inst. 1984;72:1271-1275.

23. Hyodo T, Kanazawa S, Dendo S, Kobayashi K, Hayashi H, Kouno Y, et al. Intrapulmonary lymph nodes: thin-section CT findings, pathological findings, and CT differential diagnosis from pulmonary metastatic nodules. Acta Med Okayama. 2004;58(5):235-240.

24. Choi DJ, Kwak JM, Kim J, Woo SU, Kim SH. Preoperative chest computerized tomography in patients with locally advanced mid or lower rectal cancer: its role in staging and impact on treatment strategy. J Surg Oncol. 2010;102:588-592.

25. Grossmann I, Avenarius JK, Mastboom WJ, Klaase JM. Preoperative staging with chest CT in patients with colorectal carcinoma: not as a routine procedure. Ann Surg Oncol. 2010;17:2045-2050.

26. Brent A, Talbot R, Coyne J, Nash G. Should indeterminate lung lesions reported on staging $\mathrm{CT}$ scans influence the management of patients with colorectal cancer? Colorectal Dis. 2007;9:816-818. 